\title{
Estudo das propriedades ópticas de vidros borosilicatos dopados com íons de Itérbio em função da concentração
}

\section{Study of optical properties of borosilicate glass doped with Ytterbium as a function of the concentration}

\author{
Filippe C. Bernardino ${ }^{1}$; Sidney A. Lourenço ${ }^{1 *}$; Marco A. T. da Silva ${ }^{1}$; Noelio O. \\ Dantas $^{2}$
}

\begin{abstract}
Resumo
Elementos de Terras-Raras tem sido estudados em diferentes áreas da ciência devido a suas excelentes propriedades espectroscópicas e magnéticas com possível aplicação na construção de diferentes dispositivos ópticos e elétricos (MARTINS, 2005; LOURENÇO et al., 2011). Neste trabalho, estudam-se as propriedades ópticas do íon de Itérbio $\left(\mathrm{Yb}^{3+}\right)$ inserido em uma matriz vítrea de chumbo-borosilicatos sintetizados pelo método de fusão, através das técnicas de absorção óptica e fotoluminescência. $\mathrm{O}$ íon $\mathrm{Yb}^{3+}$ foi escolhido como dopante, pois este possui um esquema de níveis de energia bastante simplificado quando comparado com os demais íons de Terras-Raras, com apenas dois estados energéticos, que o torna muito atrativo para construção de dispositivos ópticos de alta eficiência quântica. $\mathrm{O}$ aumento da temperatura de tratamento térmico assim como o aumento da concentração do íon na matriz leva a um deslocamento do gap óptico da matriz para regiões de maiores energias. Acreditamos que este deslocamento (blue-shift) pode estar associado ao processo de nanocristalização da matriz SBP $\left(\mathrm{SiO}_{2}\right.$, $\mathrm{B}_{2} \mathrm{O}_{3}, \mathrm{PbO}_{2}$ ). A redução do tempo de vida radiativo dos estados excitados do íon, em função do aumento da concentração do íon na matriz, foi analisada através da relação empírica de Stokowski, a qual, estuda processos de transferência de energia com a variação da concentração dos íons de TR
\end{abstract}

Paravras chave: Vidros de borosilicato e chumbo. Íons de Terras-Raras. Propriedades ópticas. Método de fusão.

\begin{abstract}
Rare Earth elements have been studied for different scientific areas due to its excellent spectroscopic and magnetic properties with possible application for construction of different optical and electric devices (MARTINS, 2005; LOURENÇO et al., 2011). In this work, it is studied the optical properties of Ytterbium $\left(\mathrm{Yb}^{3+}\right)$ ions embedded in a lead-borosilicate glass matrix synthesized by the melting method, using the optical absorption and photoluminescence techniques. The $\mathrm{Yb}^{3+}$ ions were chosen to dope the glass matrix because it has an energy level scheme more simplified compared with other Rare-Earth ions, with only two energy levels, making it very attractive for the construction of high efficiency optical devices. Increasing the annealing temperature as well as the ion concentration in the matrix leads to a shift of the optical band gap of the matrix to higher energies. We believe that this shift (blueshift) can be associated with the nanocrystallization process of the glass matrix $\mathrm{SBP}\left(\mathrm{SiO}_{2}, \mathrm{~B}_{2} \mathrm{O}_{3}, \mathrm{PbO}_{2}\right)$. The reduction of radiative lifetime with increasing ion concentration in matrix was studied using the Stokowski empirical relation, in which, it studies processes of energy transfer as a function of RareEarth concentration
\end{abstract}

Keywords: Lithium-ion batteries. $\mathrm{LiCoO}_{2}$ extraction. Recycling. Process optimization

1 Grupo de Síntese, Caracterização e Aplicações de Materiais Optoeletrônicos (SCAMO), Universidade Tecnológica Federal do Paraná, CEP 86036-370, Londrina, Paraná, Brasil. lourenco-sidney@hotmail.com

2 Laboratório de Novos Materiais Isolantes e Semicondutores (LNMIS), Instituto de Física, Universidade Federal de Uberlândia, CP593, 38400-902 Uberlândia, MG, Brazil. 


\section{Introdução}

As propriedades ópticas e magnéticas dos íons de Terras-Raras (TR) são extremamente interessantes e tem aplicações em variados tipos de dispositivos modernos. Catalisadores automobilísticos, laser de estado sólido, telas de aparelhos eletrônicos, imãs de alta eficiência para motores e fibras ópticas amplificadoras, estes são apenas alguns exemplos em que os elementos de Terras-Raras estão inseridos na composição de materiais com aplicações tecnológicas (MARTINS, 2005; LOURENÇO et al., 2011).

Os lantanídeos, família química na qual os elementos de Terras-Raras estão inseridos, possuem uma característica ímpar quando comparados a outros elementos da tabela periódica, chamada de contração lantanídea, que é uma diminuição do raio atômico mesmo com o aumento do número atômico. Isto ocorre devido à existência de uma blindagem eletrônica da camada $4 \mathrm{f}$ fazendo com que os elétrons adicionados sintam uma carga nuclear efetiva maior. Neste tipo de material as transições ópticas ocorrem na camada 4f, que é uma camada interna do átomo. Assim as ligações químicas do íon no meio hospedeiro, que ocorre com elétrons da camada de valência, não afetam as propriedades ópticas deste sistema, tornando-o pouco influenciado pelo meio. Graças a esta propriedade os íons de TR interagem fracamente com o campo cristalino, e assim é possível aproximar um modelo que pode descrever os níveis de energia de um íon de TR embebido em uma matriz (material que garante a estabilidade mecânica ao sistema) como o de um único íon isolado.

Para as aplicações ópticas os vidros borosilicatos são utilizados como excelente material hospedeiro, devido a suas ótimas propriedades térmicas, estabilidade química, facilidade de produção e por interferir pouco nas características ópticas dos íons de Terras-Raras (LOURENÇO et al., 2011).

O Itérbio, quando comparado com os demais íons de Terras-Raras possui um esquema de níveis de energia bastante simples, com apenas dois estados opticamente ativos na região do infravermelho próximo. Esta é uma propriedade interessante que torna este íon muito atrativo para construção de dispositivos ópticos de alta eficiência. Assim, o $\mathrm{Yb}^{3+}$ dopando uma matriz vítrea possui diversas vantagens, como a redução de processos não radiativos (HÖNNINGER et al., 1998; LIU, 1999). Além disso, os materiais dopados com $\mathrm{Yb3}+$ são de enorme interesse para a próxima geração de lasers de alta potência e também como um sensibilizador de transferência de energia para os lasers de infravermelhos por processo de up-conversion (NEES et al., 1998; DIENING; MOBERT; HUBER, 1988). Várias aplicações são relatadas para a luminescência assistidas em materiais usando $\mathrm{Yb} 3+$ como dopantes, como telas tridimensionais planas e biestabilidade intrínseca de comutação óptica (BELL et al., 2003; CACHO, et al., 2006).

Este trabalho tem por finalidade o estudo das propriedades ópticas de íons de $\mathrm{Yb}^{3+}$ inseridos em uma matriz chumbo-borosilicato, sintetizada pelo método de fusão, em diversas concentrações e tratados termicamente em duas temperaturas distintas. Para isto, utilizaram-se as técnicas de absorção óptica e fotoluminescência em amostras com diferentes concentrações do íon. Ainda foram realizadas medidas de tempo de vida com o intuito de avaliar a existência de processos não radiaativos, os quais, interferem diretamente na emissão óptica.

\section{Metodologia}

Os estudos foram realizados com base em dois conjuntos de amostras. A matriz vítrea escolhida foi uma matriz SBP, composta por $40 \mathrm{SiO}_{2} 40 \mathrm{~B}_{2} \mathrm{O}_{3}$ $20 \mathrm{PbO}_{2}$ em porcentagem molar e o íon de TerraRara como dopante desta matriz foi o Iterbio $\left(\mathrm{Yb}^{3+}\right)$, acrescentado em diferentes porcentagens como mostrado na Tabela 1 . 
Tabela 1 - Porcentagem de Íons de $\mathrm{Yb}^{3+}$ nas amostras de SBP.

\begin{tabular}{|c|c|c|c|c|c|c|c|c|}
\hline $\begin{array}{c}\text { Amostra } \\
\text { Yb }\end{array}$ & A1 & A2 & A3 & A4 & A5 & A6 & A7 & \\
\hline$\%$ & 0,1 & 0,2 & 0,3 & 0,4 & 0,5 & 1,0 & 1,5 & \\
\hline $\begin{array}{c}\text { Amostra } \\
\text { Yb }\end{array}$ & A8 & A9 & A10 & A11 & A12 & A13 & A14 & A15 \\
\hline$\%$ & 2,0 & 2,5 & 3,0 & 3,5 & 4,0 & 4,5 & 5,0 & 0,0 \\
\hline
\end{tabular}

Fonte: os autores

A matriz SBP foi produzida misturando, em um cadinho de alumina, os óxidos em pó de $40 \mathrm{SiO}_{2} 40 \mathrm{~B}_{2} \mathrm{O}_{3} 20 \mathrm{PbO}_{2}$, nessas proporções e em seguida foram fundidos a uma temperatura de aproximadamente $1300{ }^{\circ} \mathrm{C}$ durante 30 minutos. Após esta fase, a matriz foi resfriada a temperatura ambiente sobre uma placa metálica, para facilitar a troca de calor e seu manuseio. Em seguida, esta matriz foi pulverizada.

Os íons foram acrescentados na amostra em forma de óxido, $2 \mathrm{Yb}_{2} \mathrm{O}_{3}$, também em pó, em proporções segundo a Tabela 1 e fundidos novamente à $1300{ }^{\circ} \mathrm{C}$ durante de $30 \mathrm{~min}$.

As amostras, após serem resfriadas a temperatura ambiente, foram separadas em dois conjuntos de 15 amostras. O primeiro conjunto foi tratado a $350{ }^{\circ} \mathrm{C}$ por 24 horas e o segundo conjunto de amostras foi tratado em $500{ }^{\circ} \mathrm{C}$ durante 120 horas.

As temperaturas de tratamento foram escolhidas devido às propriedades térmicas da matriz vítrea, que possui temperatura de transição vítrea (Tg) em $458{ }^{\circ} \mathrm{C}$, que é uma temperatura onde inicia a mobilidade dos íons na estrutura do material e a temperatura de cristalização (Tc) por volta de $510{ }^{\circ} \mathrm{C}$, que é o ponto onde inicia o processo de cristalização na estrutura da matriz. A Figura 1 mostra um gráfico de DTA (Análise Térmica Diferencial), usada para obter a Tg e Tc da matriz.
Figura 1 - Curva de DTA da matriz vítrea de SBP, mostrando a temperatura de transição vítrea (Tg) e a temperatura de cristalização $(\mathrm{Tc})$

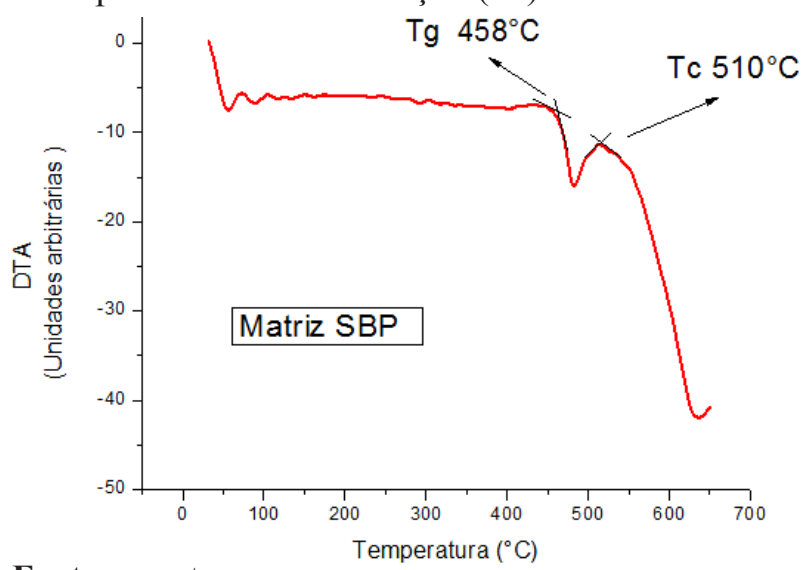

Fonte: os autores

Após o processo de síntese, as amostras, com o comprimento aproximado $10 \mathrm{~mm}$ e uma espessura de $1,5 \mathrm{~mm}$, foram polidas de forma a deixar sua superfície plana e com a menor rugosidade possível. Para isto foram usadas lixas d'água com diferentes granulações. Primeiramente foram utilizadas lixas de numeração 120 (maior granulação) para desgastar de forma intensa a superfície removendo defeitos maiores e em seguida lixas com partículas de granulometria menor, com numeração 600, 1500 e 2500, para polir as superfícies das amostras.

A Figura 2 mostra o conjunto de amostras tratadas à temperatura de $350^{\circ} \mathrm{C}$, lixadas e prontas para serem analisadas através dos métodos de caracterização óptica. 
Figura 2 - Conjunto de amostras de SBP dopadas com $\mathrm{Yb}^{3+}$, tratadas à temperatura de $350^{\circ} \mathrm{C}$.

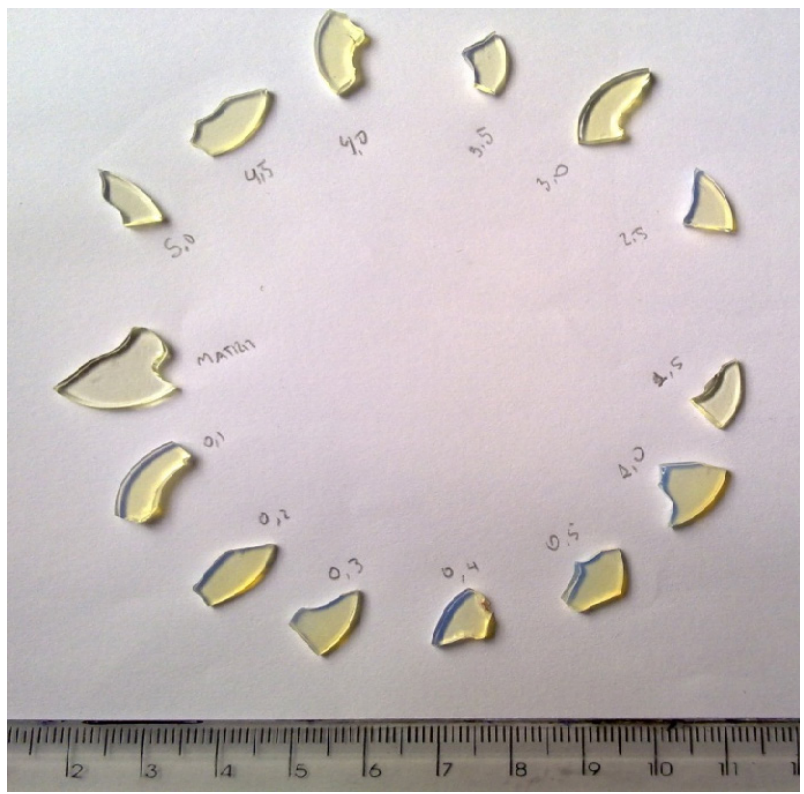

Fonte: os autores

Para realizar as medidas de absorção óptica foi utilizado o espectrofotômetro UV - visível DR5000 da marca $\mathrm{HACH}$, que trabalha em uma faixa de comprimento de onda de 190 até $1100 \mathrm{~nm}$. Sua resolução para pequenas faixas de comprimento de onda é aproximadamente $0,1 \mathrm{~nm}$. As medidas de fotoluminescência foram realizadas usando um laser de diodo, com emissão de luz polarizada, em 473 nm. A luz emitida pela amostra é selecionada por um monocromador, com comprimento focal de 0,5 metros, que em seguida é amplificada (amplificador tipo Lock-in, associado a um chopper) e por fim detectada por um fotodetector InGaAs, refrigerado termo-eletricamente. A análise do tempo de vida do estado do íon $\mathrm{Yb}^{3+}$ foi realizada usando a luz modulada por um chopper sintonizado com um osciloscópio de $40 \mathrm{MHz}$.

\section{Resultados e Discussões}

A Figura 3 mostra o espectro de absorção da amostra tratadas a 350 oC com diferentes concentrações do íon $\mathrm{Yb}^{3+}$ obtido à temperatura ambiente (295 K). Observam-se claramente duas bandas bem definidas de absorção óptica; uma com alta absorção em torno de $400 \mathrm{~nm}$ que está associada ao gap óptico da matriz e outra em torno de $975 \mathrm{~nm}$ que está associada às transições eletrônicas do íon de Terra-Rara. Para tornar mais claro a compreensão dos resultados, foram escolhidos apenas alguns valores de concentração.

Figura 3 - Gráfico referente a curvas de absorção óptica das amostras de SBP dopadas com $\mathrm{Yb}^{3+}$.

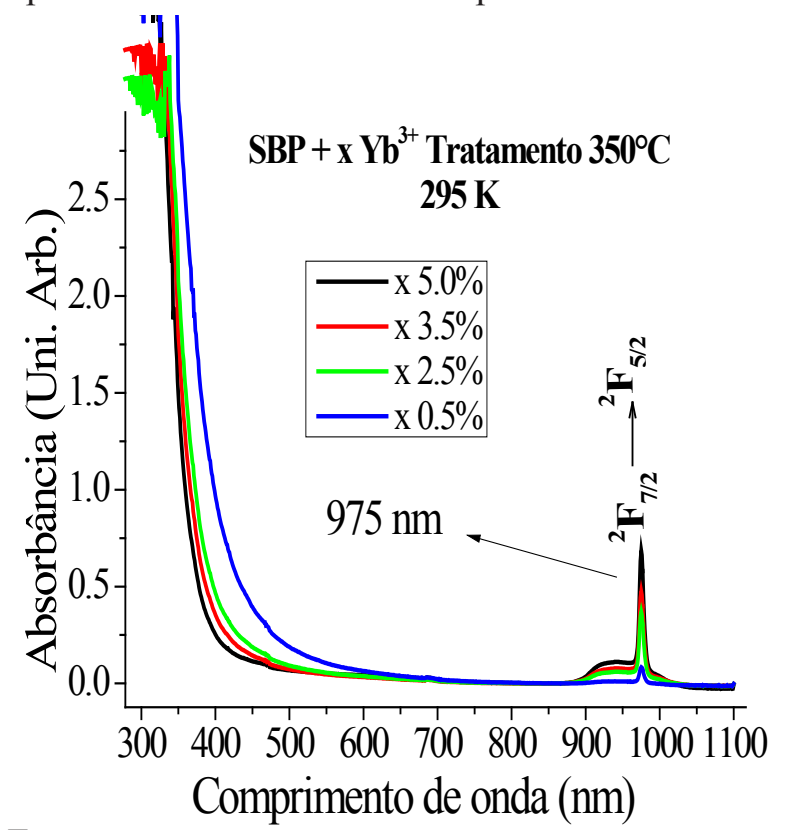

Fonte: os autores

A banda de absorção em torno de $400 \mathrm{~nm}$, referente ao gap óptico da matriz, apresenta um deslocamento para maiores energias (menores comprimentos de onda) à medida que aumenta a concentração de $\mathrm{Yb}^{3+}$. Uma forma simples de se obter o gap óptico de vidros ou cerâmicas na fase amorfa ou semicristalina é feita através do gráfico de absorbância por meio da extrapolação da reta formada no eixo de absorção ultravioleta do material com o eixo de comprimento de onda. A Figura 4 mostra o deslocamento do gap óptico para maiores energias da matriz SBP com o aumento da concentração do íon de $\mathrm{Yb}^{3+}$. Estes vidros a base de $\left(\mathrm{B}_{2} \mathrm{O}_{3}\right.$ e $\left.\mathrm{SiO}_{2}\right)$ geralmente apresentam nanocristais de $\mathrm{SiO}_{2}$ em sua estrutura (LOURENÇO et al., 2011). Estes nanocristais podem surgir a partir de tratamento térmico ou com agentes nucleantes, com alguns íons 
metálicos. Assim, íons de $\mathrm{Yb}^{3+}$ parecem facilitar o surgimento de nanocristais na matriz SBP. Fenômeno contrário foi observado recentemente para a matriz SBP dopada com íons de $\mathrm{Eu}^{3+}$ (LOURENÇO et al., 2011).

Também pode ser observado na Figura 4 que a temperatura de tratamento térmico influencia o valor do gap óptico. As amostras com tratamento térmico de $350{ }^{\circ} \mathrm{C}$ possuem menores energias de gap ópticos comparadas com aqueles das amostras tratadas a $500{ }^{\circ} \mathrm{C}$, indicando que houve uma mudança na rede cristalina do material.

Figura 4 - Dependência das energias do gap óptico em função da concentração de SBP xYb3+ das amostras de SBP com tratamentos térmicos de 350 ${ }^{\circ} \mathrm{C}$ e $500{ }^{\circ} \mathrm{C}$.

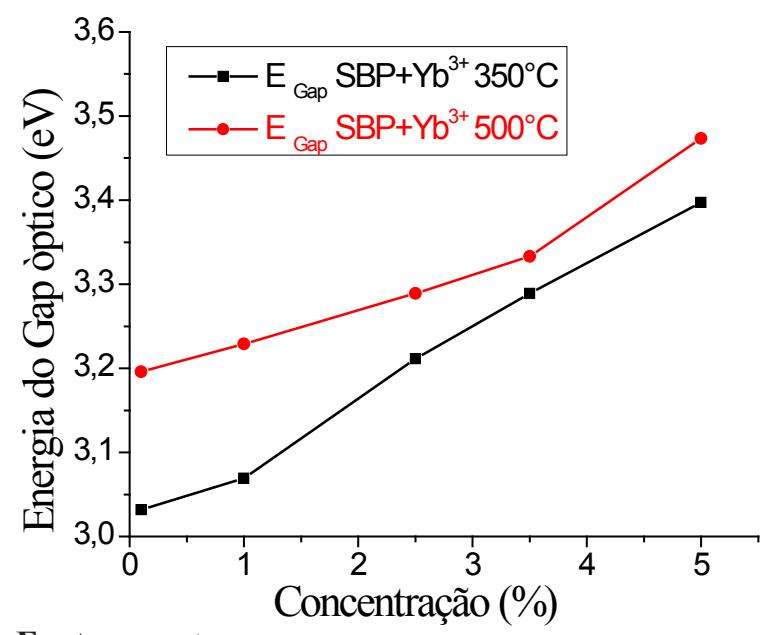

Fonte: os autores

Por outro lado, para a matriz SBP dopada com Eu3+ foi observado recentemente que o gap óptico diminui o valor de energia com o aumento da temperatura de tratamento térmico (LOURENÇO et al., 2011), efeito associado a uma inibição da cristalização da matriz. Estudos mais sistemáticos devem ser realizados para explicar por que o grau de cristalização se elava com o aumento da temperatura de tratamento térmico na amostra SBP com $\mathrm{Yb}^{3+}$.

A absorção por volta de 975 nmé uma característica dos íons de $\mathrm{Yb}^{3+}$ (SILVA, 2006) e está associada à transição óptica entre dois estados eletrônicos, o estado
${ }^{2} \mathrm{~F}^{7 / 2}$ e o estado ${ }^{2} \mathrm{~F}^{5 / 2}$. Com o aumento da concentração do dopante, os picos de absorbância ficam cada vez mais intensos, indicando que os íons de $\mathrm{Yb}^{3+}$ foram efetivamente incorporados à matriz hospedeira.

AFigura 5 mostra os espectros de fotoluminescência obtidos à temperatura ambiente da amostra SBP tratada a $350{ }^{\circ} \mathrm{C}$ para diferentes concentrações de $\mathrm{Yb}^{3+}$. Com o aumento da concentração ocorre um aumento gradativo da intensidade da luminescência similar ao observado nos espectros de absorção óptica. Comportamento semelhante foi observado para a amostra tratada a $500{ }^{\circ} \mathrm{C}$.

A Figura 6 exibe um diagrama de energia com os dois estados degenerados do íon $\mathrm{Yb}^{3+}$; o estado ${ }^{2} \mathrm{~F}^{5 / 2}$ e ${ }^{2} \mathrm{~F}^{7 / 2}$ (SERQUEIRA, 2010). As setas pretas para cima indicam a excitação eletrônica quando ocorre à absorção de fótons onde a seta mais espessa representa a absorção do pico de 975 nm (DAÍ; HU, 2003) e as demais, de menor espessura, representam o ombro próximo ao pico que está entre 900 e $960 \mathrm{~nm}$.

As setas azuis, para baixo, representam a emissão de fótons do estado ${ }^{2} \mathrm{~F}_{5 / 2}$ para o estado ${ }^{2} \mathrm{~F}_{7 / 2}$, onde a seta azul, de maior espessura, está relacionada ao pico de emissão do $\mathrm{Yb}^{3+}$ em $978 \mathrm{~nm}$ e, as demais setas, com o ombro da emissão de 990 a $1060 \mathrm{~nm}$.

Figura 5 - Espectro de emissão, provido pela técnica de fotoluminescência. Amostras de SBP tratadas a $350{ }^{\circ} \mathrm{C}$, colocando apenas um estado eletrônico, variando a concentração de $\mathrm{Yb}^{3+}$, a temperatura ambiente.

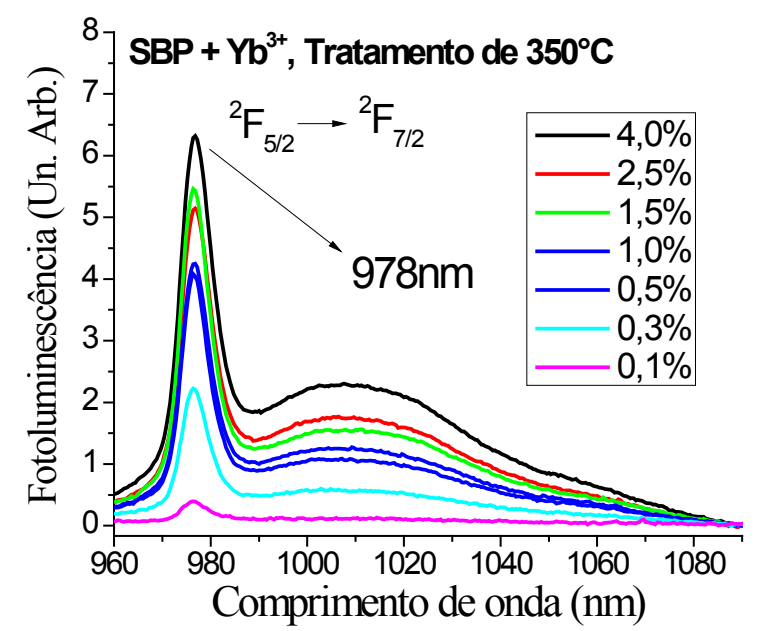

Fonte: os autores 
Figura 6 - Diagrama de níveis de energia do $\mathrm{Yb}^{3+}$ em uma matriz SBP. As setas para cima indicam a excitação dos elétrons do estado fundamental ${ }^{2} \mathrm{~F}_{7 / 2}$ para a o estado excitado ${ }^{2} \mathrm{~F}_{5 / 2}$. (Absorção). As setas para baixo indicam a transição de elétrons do estado excitados para o estado fundamental, emitindo fótons.

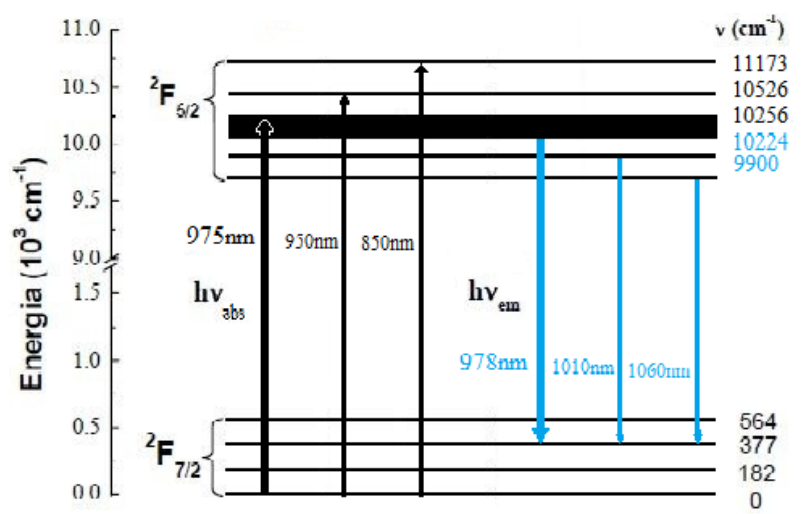

Fonte: os autores

A Figura 7 mostra um comparativo entre as curvas de absorção óptica e fotoluminescência nas duas temperaturas de tratamento térmico $\left(350{ }^{\circ} \mathrm{C}\right.$ e $500{ }^{\circ} \mathrm{C}$ ). É possível visualizar dois ombros nas curvas de emissão e absorção próximas aos picos centrais, em $975 \mathrm{~nm}$ e $978 \mathrm{~nm}$. O primeiro ombro localizado antes do pico de $975 \mathrm{~nm}(900 \mathrm{~nm}$ a $960 \mathrm{~nm}$ ) representa a absorção do nível eletrônico ${ }^{2} \mathrm{~F}_{5 / 2}$ perturbado pelo campo cristalino da matriz. O segundo ombro localizado após o pico de emissão em $978 \mathrm{~nm}(990 \mathrm{~nm}$ até $1060 \mathrm{~nm})$, está associada ao mesmo estado ${ }^{2} \mathrm{~F}_{5 / 2}$, perturbado pelo campo cristalino da amostra.

Nesta figura, observa-se que as emissões dos fótons, do estado ${ }^{2} \mathrm{~F}_{5 / 2}$ possuem menor energia $\left(10224 \mathrm{~cm}^{-1}\right)$ comparado com a absorção de fótons do mesmo estado $\left(10256 \mathrm{~cm}^{-1}\right)$. Essa diferença, que corresponde a um valor de $6,4 \mathrm{meV}$, é um valor esperado e conhecido na literatura como Stoke-Shift. Este valor é relativamente pequeno e está associado à baixa interação do íon com a matriz.
Figura 7 - Espectros de absorbância óptica (vermelha e preta) e fotoluminescência (azul e verde) da matriz SBP dopada com $4 \%{\mathrm{de} \mathrm{Yb}^{3+}}^{3+}$ tratadas a $350{ }^{\circ} \mathrm{C}$ e $500{ }^{\circ} \mathrm{C}$.

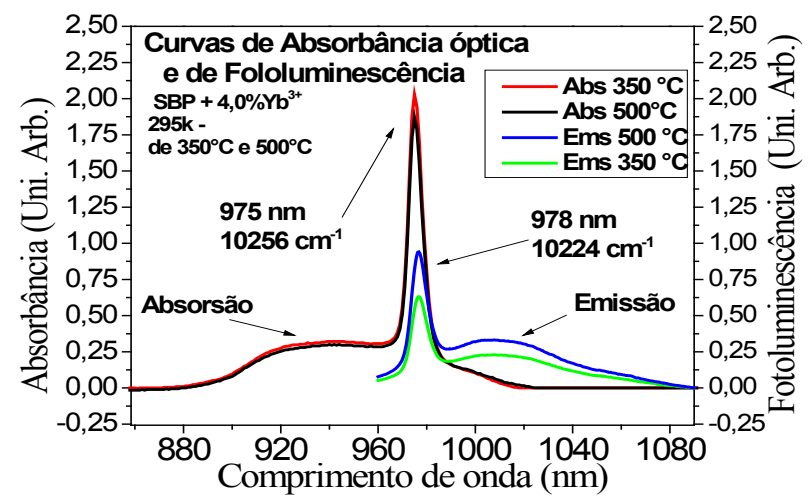

Fonte: os autores

A Figura 8 mostra um aumento gradativo da área abaixo da curva dos picos principais dos espectros de absorção e luminescência em função do aumento da concentração do íon, com uma tendência de saturação para altas concentrações. Esta tendência provavelmente está associada à presença de mecanismos não radiativos, para altas concentrações do íon, que competem com as transições radiativas (SILVA, 2006).

Figura 8 - Área integrada do pico, mais intenso, dos espectros de luminescência e absorção em função da concentração dos íons na amostra tratada $350{ }^{\circ} \mathrm{C}$.

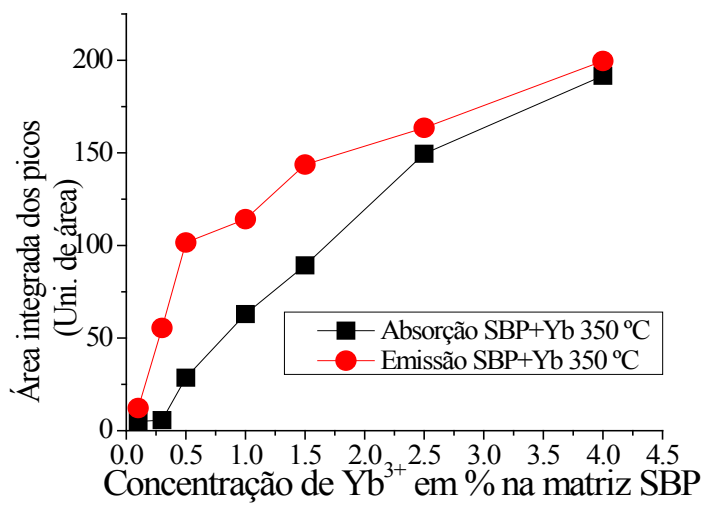

Fonte: os autores 
A Figura 9 exibe uma curva do tempo de vida de decaimento do estado excitado ${ }^{2} \mathrm{~F}_{5 / 2}$ do íon $\mathrm{Yb}^{3+}$ em função da concentração à temperatura ambiente. O decaimento do sinal de PL foi ajustado por uma função monoexponencial decrescente, representada pelos pontos circulares na Figura 9, indicando a presença de apenas um único canal de decaimento não radiativo.

Figura 9 - Curvas de intensidade de sinal de fotoluminescência em função do tempo para as amostras de SBP dopadas com $\mathrm{xYb}^{3+0} \%$. Os círculos representam a ajuste de uma função monoexponencial aos dados experimentais.

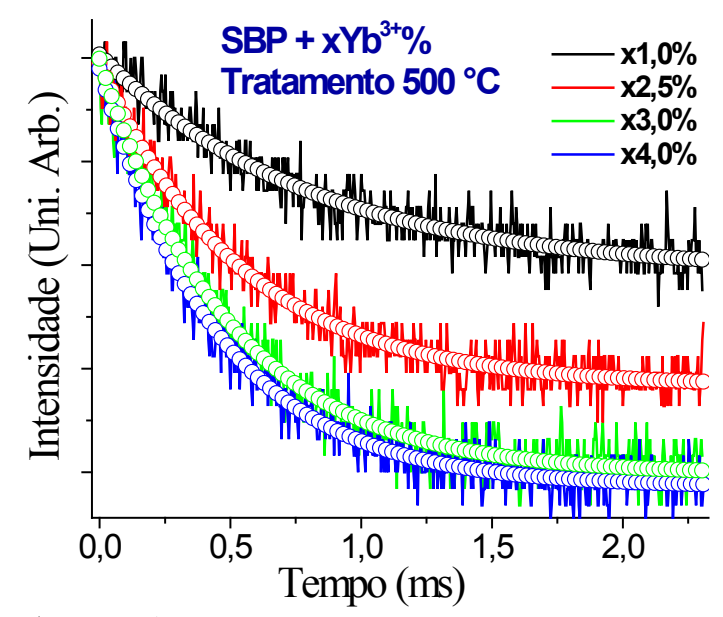

Fonte: os autores

O tempo de decaimento radiativo $(\tau=1 / \mathrm{e})$, obtido do ajuste, é de 1,0 ms para baixas concentrações do íon sofrendo uma forte redução com o aumento da concentração como pode se observar na Figura 10.
Figura 10 - Gráfico do tempo de vida em função da concentração de íons de $\mathrm{Yb}^{3+}$, ajustado pela relação de Stokowski. Os círculos pretos indicam os pontos experimentais e a curva em vermelho o ajuste usando a Eq. (1).

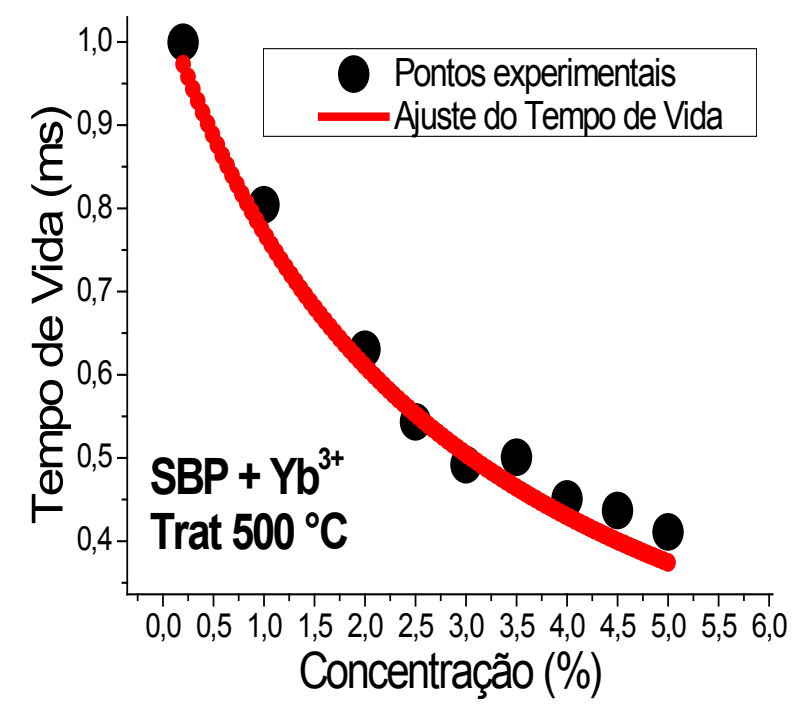

Fonte: os autores

Sabe-se que os processos de decaimento não radiativos, reduzem o tempo de vida do portador no estado excitado. Estes processos são regidos por transferência de energia dos íons de TR para o material hospedeiro. Exemplos de centros com transição não radiativos são: modos vibracionais de rede por carbonilas e hidroxilas e processo de relaxação cruzada (interação íon-íon) (BELL et al., 2003). Os baixos valores de tempo de vida do estado ${ }^{2} \mathrm{~F}_{5 / 2}$ para altas concentrações de $\mathrm{Yb}^{3+}$ ocorre 
provavelmente devido à migração de energia entre os íons excitados de $\mathrm{Yb}^{3+}$ e a transferência de energia envolvendo impurezas ou defeitos próximos aos íons de $\mathrm{Yb}^{3+}$.

Stokowski (ver ref. DIGONNET, 2001) estudaram os processos de transferência de energia dos íons de $\mathrm{Nd}^{3+}$ e estabeleceram uma relação empírica, (equação 1) entre o tempo de vida e a concentração dos íons de TR.

$$
\tau_{\text {exp }}=\frac{\tau_{0}}{1+(N / Q)^{n}},
$$

Onde $\tau 0$ é o tempo de vida observado para um sistema diluído (baixa concentração de íons de TR), Q é a concentração do íon quando o tempo de vida se reduz pela metade, $\mathrm{N}$ é o valor da concentração e $n$ é o fator que informa o tipo de mecanismo de decaimento eletrônico.

É estabelecido na literatura científica que valores de $\mathrm{n}$ próximos a 2 , sugerem um sistema ressonante, governado por mecanismos de relaxação cruzada. (SERQUEIRA, 2010). Após ajuste da equação (1) aos dados experimentais, obtivemos o valor de $n=1,018 \pm 0,084$ sugerindo um mecanismo não ressonante de decaimento, processo de não relaxação cruzada. Provavelmente a redução do tempo de vida, possa estar relacionado com a presença de hidroxilas OH- (CAETANO, 2013). Medidas recentes de absorção no infravermelho nesta matriz (SBP), mostram a presença da vibração de hidroxilas OH- (LOURENÇO et al., 2013). Com o aumento da concentração, os íons de $\mathrm{Yb}^{3+}$ tendem a ficar mais próximos uns dos outros, permitindo uma migração dos elétrons de um íon para o outro antes do processo de recombinação efetivamente ocorrer. Este processo de migração aumenta a probabilidade do elétron encontrar um defeito na estrutura da matriz recombinando-se não radiativamente ou ficar aprisionado neste defeito reduzindo, dessa forma, o tempo de vida do estado.

\section{Conclusões}

Neste trabalho foi analisado as propriedades ópticas do íon Terra-Rara $\mathrm{Yb}^{3+}$ inserido na matriz SBP $\left(40 \mathrm{SiO}_{2} \quad 40 \mathrm{~B}_{2} \mathrm{O} 3 \quad 20 \mathrm{PbO}_{2}\right)$ em função da concentração do íon e da temperatura de tratamento térmico da matriz. As temperaturas de transição vítrea e de cristalização da matriz foram obtidas pela análise da curva de DTA, cujos valores obtidos foram: $\mathrm{Tg}=458{ }^{\circ} \mathrm{C} \mathrm{e} \mathrm{Tc}=510^{\circ} \mathrm{C}$, respectivamente.

O espectro de absorção óptica apresenta um pico em $975 \mathrm{~nm}$, relativo à transição eletrônica do estado ${ }^{2} \mathrm{~F}_{7 / 2}$ para ${ }^{2} \mathrm{~F}_{5 / 2}$, característica do íon Yb3+. Observando os espectros de absorção óptica pode-se concluir que à medida que se aumenta a concentração de $\mathrm{Yb}^{3+}$, ocorre um aumento da intensidade da absorção proporcional a incorporação do íon na matriz.

O aumento da concentração do íon leva a um deslocamento do gap óptico para maiores energias. Este comportamento também foi observado ao aumentar a temperatura de tratamento térmico de $350{ }^{\circ} \mathrm{C}$ para $500{ }^{\circ} \mathrm{C}$, e estão associados ao processo de cristalização da matriz. Relatos da literatura científica mostram que vidros borosilicatos geralmente apresentam nanocristalização de $\mathrm{SiO}_{2}$ (LOURENÇO et al., 2011). Neste trabalho verificamos que os íons de $\mathrm{Yb}^{3+}$ podem ser usados como facilitadores da cristalização de nanocristais de $\mathrm{SiO}_{2}$ quando incorporados na matriz SBP.

Os espectros de luminescência mostram um pico bem definido com emissão em $978 \mathrm{~nm}$ indicando um decaimento eletrônico do nível de maior energia ${ }^{2} \mathrm{~F}_{5 / 2}$ para o estado de menor energia ${ }^{2} \mathrm{~F}_{7 / 2}$. Ao comparar os dois picos tanto de absorção quanto de emissão encontra-se uma pequena diferença de energia 6,4 meV, conhecida como Stoke-Shift. Este baixo valor está associado à baixa interação do íon com a matriz.

Uma análise da fotoluminescência com resolução temporal mostrou que o decaimento radiativo do estado ${ }^{2} \mathrm{~F}_{5 / 2}$ do íon $\mathrm{Yb}^{3+}$, na matriz SBP, pode ser ajustado com uma única função exponencial 
decrescente de onde obteve-se um tempo de vida de 1,0 ms para a amostra com menor concentração de $\mathrm{Yb}^{3+}$. Com o aumento da concentração dos íons de $\mathrm{Yb}^{3+}$, o tempo de vida do estado sofreu uma sensível redução que pode estar associada à presença de hidroxilas na matriz.

\section{Referências}

BELL, M. J. V.; QUIRINO, W. G.; OLIVEIRA, S. L.; SOUSA, D. F.; NUNES, L. A. O. Cooperative luminescence In $\mathrm{Yb}^{3+}$ doped phosphate glasses. Journal of Physicsv: Condensed Matter, Bristol, v. 15, p. 4877, 2003.

CACHO, V. D.; KASSAB, L. R. P.; OLIVEIRA. S. L.; MANSANO, R. D.; VERDONCK, P. Blue cooperative luminescence properties in $\mathrm{Yb}^{3+}$ doped $\mathrm{GeO}_{2}-\mathrm{PbO}-\mathrm{Bi}_{2} \mathrm{O}_{3}$ vitreous system for the production of thin films. Thin solid films, Amsterdam, v. 515, n. 2, p. 764-767, 2006.

CAETANO, M. Estudo de sistemas vitreos dopados com $\mathrm{Er}^{3+}$ visando aplicações em fibras e amplificadores ópticos. 2013. Dissertação (Mestrado em Física) - Universidade Federal de Uberlândia, Uberlândia.

DAI, N.; HU, L. Effect of Yb ion concentration on the spectral proprieties of lead silica glasses. Optics Communications, Amsterdam, v. 253, p. 151-155, 2003.

DIENING, A.; MOBERT, P. E. A.; HUBER, G. J. Diode-pumped continuous-wave, quasicontinuous-wave, and Q-switched laser operation of $\mathrm{Yb}^{3+}, \mathrm{Tm} 3+$ : YLiF4 at 1.5 and 2.3 $\mu \mathrm{m}$. Journal of Applied Physics, New York, v. 84, n. 11 , p. 5900-5904, 1998.

DIGONNET, M. J. F. Rare-earth-doped fiber lasers and amplifiers, revised and expanded. Boca Raton: CRC press, 2001.

HÖNNINGER， C.; MORIER-GENOUD, F.; MOSER, M.; KELLER, U.; BROVELLI, L. R.; HARDER. C. Efficient and tunable diodepumped femtosecond $\mathrm{Yb}$ : glass lasers. Optics Letter, New York, v. 23, n. 2, p. 126-128, 1998.
LIU, H.; BISWAL, S.; PAYE, J.; NEES, J.; MOUROU, G.; HÖNNINGER, C.; Keller, U. Directly diode-pumped millijoule subpicosecond $\mathrm{Yb}$ :glass regenerative amplifier Keller. Optics Letters, New York, v. 24, n. 13, p. 917-919, 1999.

LOURENÇO, S. A.; DANTAS, N. O.; SERQUEIRA, E. O.; AYTA, W. E. F.; ANDRADE, A. A.; FILADELPHO, M. C.; SAMPAIO, J. A.; BELL, M. J. V.; SILVA, M. A. P. $\mathrm{Eu}^{3+}$ photoluminescence enhancement due to termal energy transfer in $\mathrm{Eu}_{2} \mathrm{O}_{3}$ - doped $\mathrm{SiO}_{2}-\mathrm{B}_{2} \mathrm{O}_{3}-\mathrm{PbO}_{2}$ glasses system. Journal of Luminescence, Amsterdam, v. 131, n. 5, p. 850855, 2011.

LOURENÇO, S. A.; LAURETO, E.; ANDRADE, A. A.; SILVA, A. C. A.; DANTAS, N. O. Efficient energy transsfer mediated by intrisic $\mathrm{SiO} 2$ nanocrystals in $\mathrm{Eu}^{+3}$ - doped lead borosilicate glasses. Materials chemistry and physics, Amsterdam, v. 139, n. 2/3, p. 471-477, 2013.

MARTINS, T. S. Terras raras: aplicações industriais e biológicas. Química Nova, São Paulo, v. 28, n. 1, p.111-117, 2005.

NEES, J.; BISWAL, S.; DRUON, F.; FAURE, J.; NANTEL, M.; MOUROU, G. A.; NISHIMURA, A.; TAKUMA, H.; ITATANI, J.; CHANTELOUP, J. C.; HONNINGER, C.; IEEE, J. S. Quarter-Wave discrete wavelet design of dichroic highly reflecting-transmitting mirrors for ultrafast solid-state lasers. Applied optics, Washington, DC, v. 6, p. 1261-1265, 2004.

HENDERSON, N.; THURSTON, J.; GSCHNIDNER, K. A.; Rare earths: crucial elements of advanced technologies. Material Matters, Milwaukee, v. 6, n. 2, p. 31-58, 2011.

SERQUEIRA, E. O. Estudo de parâmetros espectroscópicos de ions de $\mathrm{Nd}^{3+}$ nos sistemas vitreos $\mathrm{SNAB}\left(\mathrm{SiO}_{2}-\mathrm{Na}_{2} \mathrm{O}_{3}-\mathrm{Al}_{2} \mathrm{O}_{3}-\mathrm{B}_{2} \mathrm{O}_{3}\right)$ nanestruturados com nanocristais de $\mathrm{C} d S$. 2010. Tese (Doutorado em Física da Matéria condensada) - Universidade Federal de Uberlândia, Uberlândia. 
SILVA, C. J. Caracterização termo-óptica de materiais lasers usando a técnica de lente térmica. 2006. Tese (Doutorado em Ciência Física Aplicada) - Universidade de São Paulo, São Carlos. 